Paper No. 13

$12^{\text {th }}$ Intern. Cryocooler Conf.

June 18-20, 2002

Boston, MA

\title{
Low Temperature Cryocooler Regenerator Materials
}

\author{
K.A. Gschneidner, Jr. ${ }^{1,2}$, A.O. Pecharsky ${ }^{1}$, and V.K. Pecharsky ${ }^{1,2}$ \\ ${ }^{1}$ Ames Laboratory and ${ }^{2}$ Department of Materials Science and Engineering \\ Iowa State University, Ames, Iowa 50011-3020, USA
}

\begin{abstract}
There are four important factors which influence the magnitude of the magnetic heat capacity near the magnetic ordering transition temperature. These include the theoretical magnetic entropy, the deGennes factor, crystalline electric field, and the RKKY (RudermanKittel-Kasuya-Yosida) interaction. The lattice contribution to the heat capacity also needs to be considered since it is the sum of the lattice and magnetic contributions which give rise to the heat capacity maxima. The lattice heat capacity depends on the chemical composition, crystal structure and temperature. As a result, one can obtain large changes in the heat capacity maxima by alloying. Several ternary intermetallic systems have been examined in light of these criteria. A number of deviations from the expected behaviors have been found and are discussed.
\end{abstract}

\section{INTRODUCTION}

The use of lanthanide materials as low temperature regenerators has led to some significant advances in low temperature $(<20 \mathrm{~K})$ cryogenics since 1990 . The first material to be utilized was $\mathrm{Er}_{3} \mathrm{Ni}$, which replaced $\mathrm{Pb}$ in the low temperature stage of a two-stage Gifford-McMahon (G-M) cryocooler. ${ }^{1,2}$ This modification enabled Toshiba scientists to lower the low temperature limit of the G-M cryocooler from $\sim 10 \mathrm{~K}$ to $\sim 4 \mathrm{~K}$. Subsequently, several other lanthanide materials, in particular $\mathrm{Nd}^{3}$ and $\mathrm{HoCu}_{2}$ (Ref. 4), have been utilized for cooling down to $\sim 4 \mathrm{~K}$. More recently Er and Er-Pr alloys (up to 50 at.\% Pr) have been suggested as a replacement for $\mathrm{Pb}^{5,6}$ as the intermediate temperature $(\sim 10$ to $\sim 60 \mathrm{~K})$ range regenerator material. Today research is still being continued on finding improved regenerator materials, especially below $10 \mathrm{~K}$, in order to enhance the performance of Stirling, G-M, and pulse tube cryocoolers. In this quest for new and better magnetic regenerator materials there are no predictive theories or first principles computational method which allows one to foretell the composition of an alloy or intermetallic compound with the desired thermal properties, i.e. magnetic ordering temperature and total heat capacity. There are, however, a few guiding principles which can be utilized to find such materials, and these are described below.

\section{GUIDING PRINCIPLES}

\section{Magnetic Entropy}

The high heat capacity of a magnetic lanthanide material is strongly dependent on the magnetic entropy associated with the magnetic ordering process. The theoretical molar magnetic 


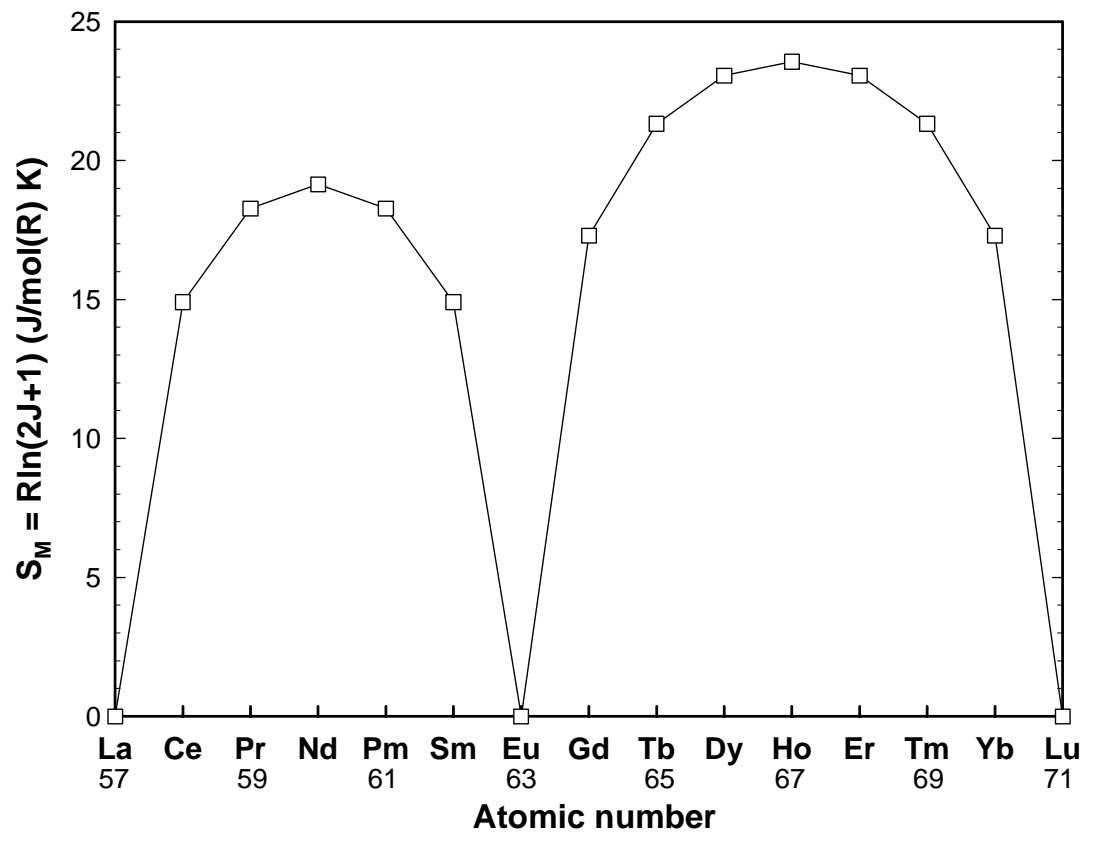

Figure 1. The molar magnetic entropy of the trivalent lanthanide elements as a function of the atomic number.

entropy, $\mathrm{S}_{\mathrm{M}}$, is proportional to the total orbital quantum number $\mathrm{J}$ and is given by

$$
\mathrm{S}_{\mathrm{M}}=\mathrm{R} \ln (2 \mathrm{~J}+1),
$$

where $\mathrm{R}$ is the gas constant. A plot of $\mathrm{S}_{\mathrm{M}}$ vs. the lanthanide atomic number is shown in Fig. 1 . There are two maxima: the minor one in the middle of the light lanthanides centered around $\mathrm{Nd}$, and the major one in the middle of the heavy lanthanides centered around Ho.

The magnetic entropy is divided up among several processes which may occur in these elements. Generally, the major one is the consumption of $\mathrm{S}_{\mathrm{M}}$ by the magnetic ordering process(es). Many times, however, lanthanide materials will exhibit more than one kind of magnetic ordering, and so, $S_{M}$ will be divided among them. Usually this is unfortunate for cryocooler applications, especially if the two ordering temperatures are far apart, since some of the entropy will not be available at the desired temperature. Sometimes this may be useful if the two magnetic ordering processes occur only a few degrees apart, such as in $\mathrm{HoCu}_{2}$, see Fig. 2. Also shown in Fig. 2 is the volumetric heat capacity of $\mathrm{Er}_{3} \mathrm{Ni}$ which exhibits only one magnetic transition. As one can see the maximum value of the heat capacity is nearly the same for the two

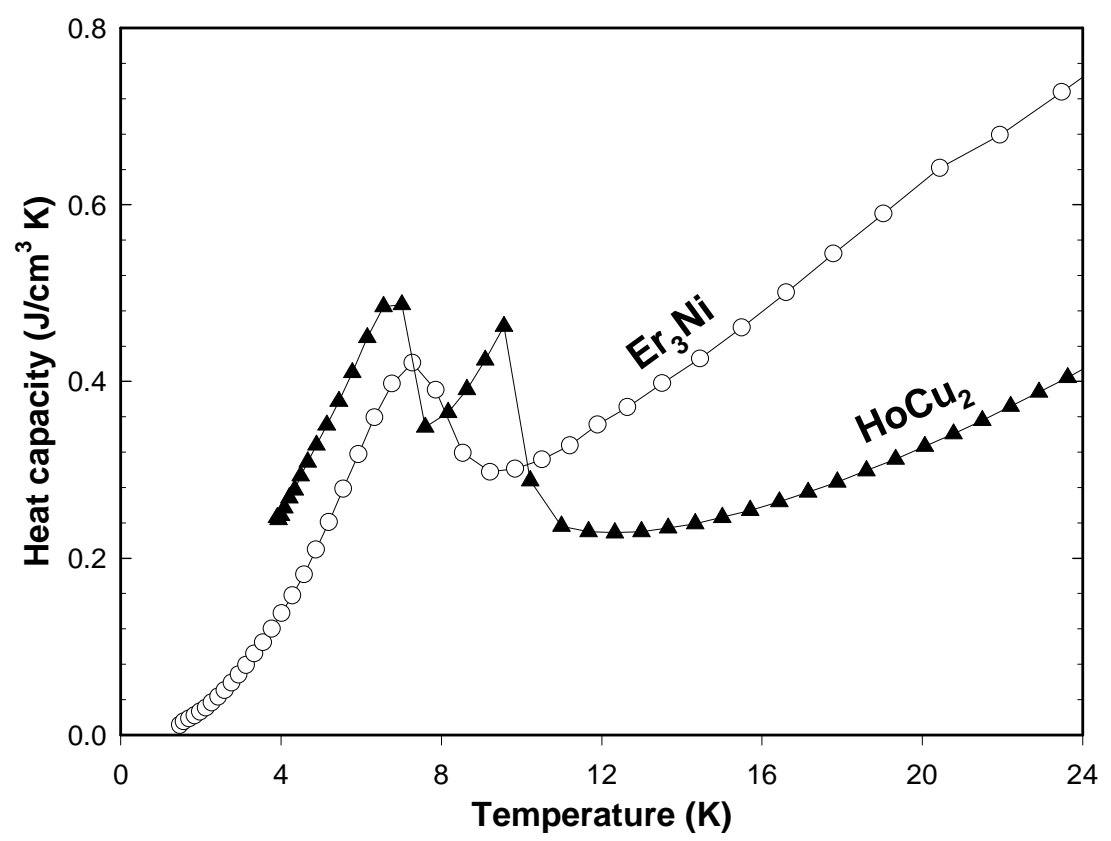

Figure 2. Volumetric heat capacity of $\mathrm{Er}_{3} \mathrm{Ni}$ and $\mathrm{HoCu}_{2}$ from $\sim 1.5$ and $\sim 3 \mathrm{~K}$, respectively, to $24 \mathrm{~K}$. 
$\mathrm{HoCu}_{2}$ peaks and about $10 \%$ larger than that of the $\mathrm{Er}_{3} \mathrm{Ni}$ peak. But for $\mathrm{HoCu}_{2}$ the heat capacity which is greater than $0.32 \mathrm{~J} / \mathrm{cm}^{3} \mathrm{~K}$ occurs over a wider temperature span $(\sim 5$ to $\sim 10 \mathrm{~K})$ than for $\mathrm{Er}_{3} \mathrm{Ni}(\sim 6$ to $\sim 8.5 \mathrm{~K})$, and therefore, because of these two reasons $\mathrm{HoCu}_{2}$ is a better regenerator for cooling below $10 \mathrm{~K}$. As a matter of fact $\sim 90 \%$ of the $<10 \mathrm{~K}$ cryocoolers utilize $\mathrm{HoCu}_{2}$ as the regenerator material. ${ }^{7}$

Spin fluctuations of the magnetic $4 f$ electrons above the highest magnetic ordering temperature generally consume about $15 \%$ of $\mathrm{S}_{\mathrm{M}}$. Thus, one would expect that about $85 \%$ of the magnetic entropy is associated with the magnetic ordering process(es). ${ }^{8}$ But crystalline electric field effects, see next subsection, also compete for this entropy, and thus could lead to a reduction in the magnetic heat capacity at the ordering temperature.

\section{Crystalline Electric Field (CEF)}

When a lanthanide ion is placed in a solid, the $4 f$ levels are split by the electric point charges of the surrounding atoms (both lanthanide and non-lanthanide). A generalized CEF energy level scheme is shown in Fig. 3 for the trivalent lanthanides. The actual energy levels will vary considerably from compound to compound depending upon the electric point charges and the point symmetry around a specific lanthanide ion. But for a given isostructural compound series, in which only the lanthanide atom varies, the relative crystal field levels across the lanthanide series will have a similar pattern, as shown in Fig. 3 for the cubic case. For a different compound series the energy scale may shift as much as an order of magnitude or more.

The entropy utilization occurs as a sample is warmed because the $4 f$ electrons in the lowest level are thermally excited to higher CEF levels. This process shows up as a broad bump in the heat capacity and is known as a Schottky anomaly. In Fig. 4 we compare the broad Schottky heat capacity anomaly for $\mathrm{Er}_{3} \mathrm{Ni}^{9}$ with the very sharp magnetic ordering peak of HoSb. ${ }^{10}$ The Schottky anomaly in $\mathrm{Er}_{3} \mathrm{Ni}$ accounts for the large heat capacity above the magnetic ordering peak in $\mathrm{Er}_{3} \mathrm{Ni}$, see Fig. 2, where it is significantly larger than that of $\mathrm{HoCu}_{2}$ above $10 \mathrm{~K}$ (and about equal to that of $\mathrm{Pb}$ up to $\sim 20 \mathrm{~K}$ ). As noted by Takahashi et al. ${ }^{9}$ about $75 \%$ of the entropy is utilized in thermal excitations of the $4 f$ electrons from low CEF levels to higher ones, while only $25 \%$ is involved in the magnetic ordering of the Er $4 f$ electrons in $\mathrm{Er}_{3} \mathrm{Ni}$.

\section{Magnetic Ordering Temperature}

The magnetic ordering temperature in a given lanthanide compound series tend to follow the deGennes factor, $\mathrm{F}$,

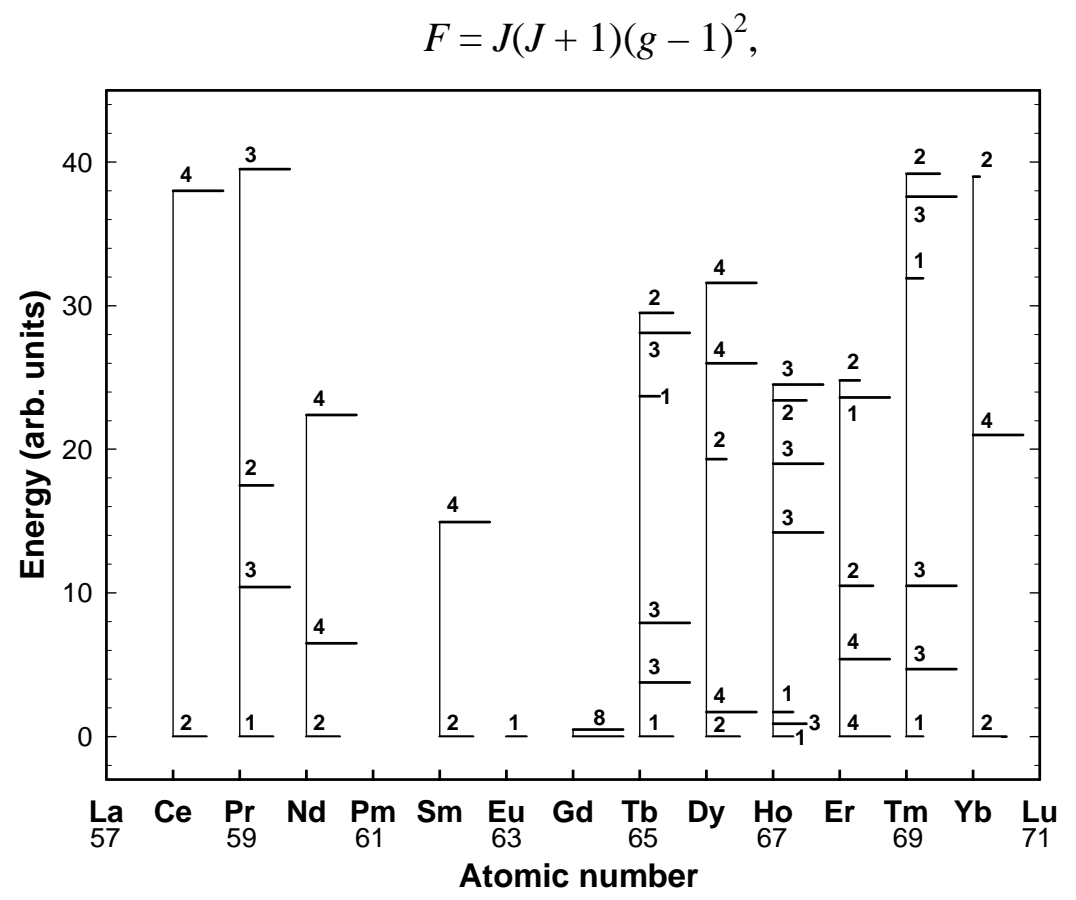

Figure 3. The crystalline electric field splittings for the trivalent lanthanide ions in a cubic environment. The numbers above or to the right of the energy level indicate the degeneracy of the level. 


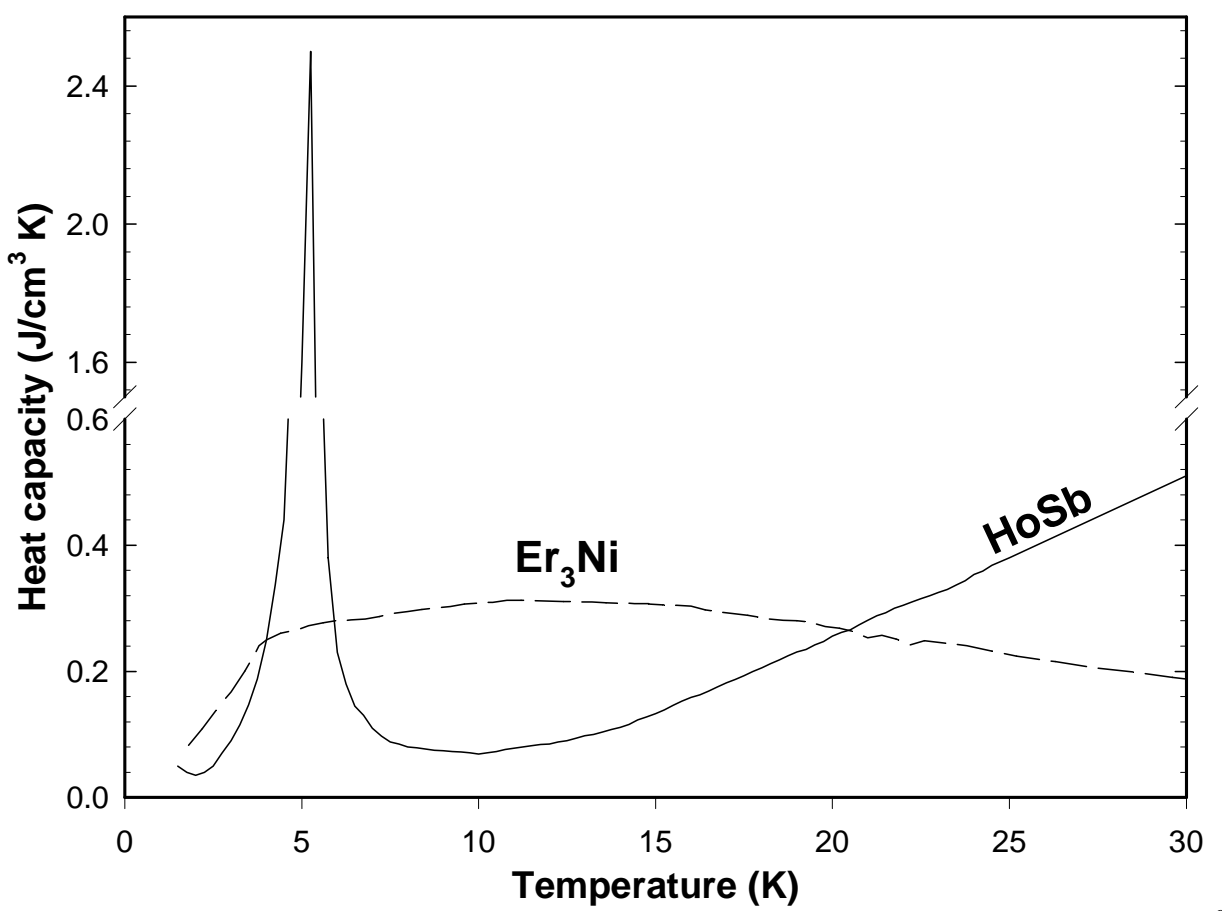

Figure 4. Volumetric Schottky heat capacity of $\mathrm{Er}_{3} \mathrm{Ni}$ due to crystalline electric field effect ${ }^{9}$ and the total volumetric heat capacity of HoSb. ${ }^{10}$

where $\mathrm{g}$ is the gyromagnetic ratio and is given by

$$
g=1+\frac{J(J+1)+S(S+1)-L(L+1)}{2 J(J+1)},
$$

where $\mathrm{S}$ is the spin quantum number and $\mathrm{L}$ is the orbital angular momentum quantum number. The variation of the deGennes factor as a function of the lanthanide atomic number is shown in Fig. 5. It should be mentioned that CEF effects can cause anomalies in the sequence of magnetic ordering temperatures expected from the deGennes factor. For example, $\mathrm{NdGe}$ is expected to have a higher Curie (ferromagnetic ordering) temperature than PrGe (see Fig. 5), but in fact, $\mathrm{NdGe}$ orders at $18 \mathrm{~K}$ while PrGe orders at $30 \mathrm{~K}$. ${ }^{11}$ As a whole, the deGennes factor is a fairly good guide for estimating the ordering temperatures in a given lanthanide compound series if

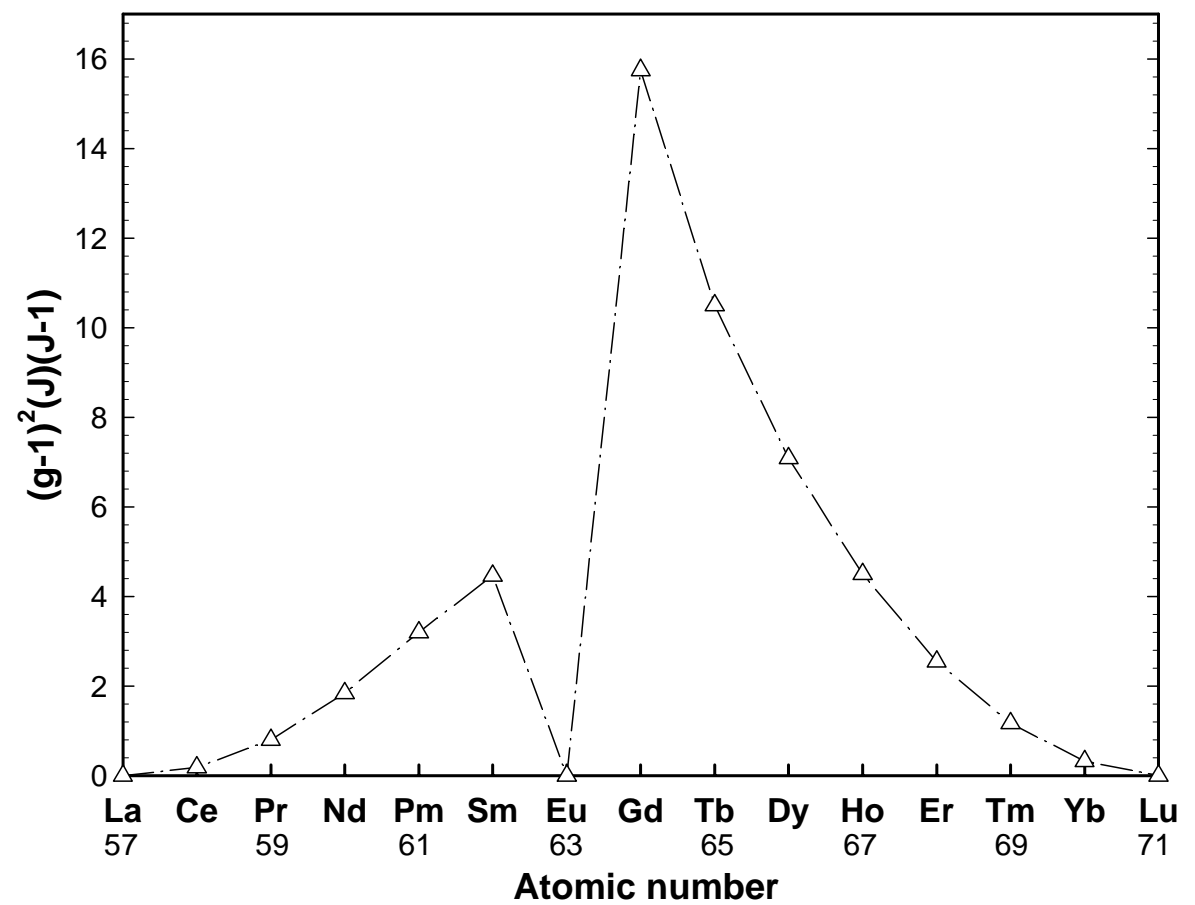

Figure 5. The deGennes factor for the lanthanide series. 


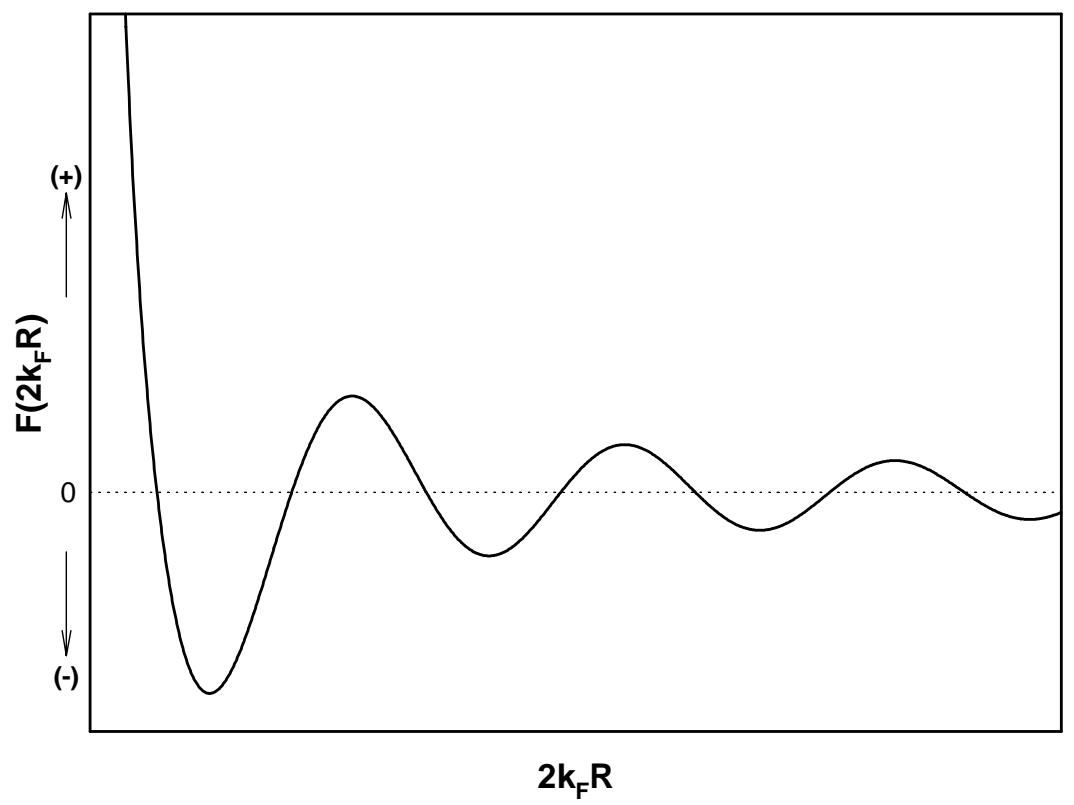

Figure 6. The spatial extent of the conduction electron polarization produced by a $4 f$ ion located at the origin $(\mathrm{R}=0)$.

one (or two) of the magnetic ordering temperatures is(are) known. In general the deGennes factor cannot be used to estimate ordering temperatures for a given $\mathrm{RM}_{\mathrm{x}}$ compound series from another $\mathrm{RN}_{\mathrm{y}}$ series.

\section{The RKKY Interaction}

Since there is little if any direct overlap of $4 f$ electron wave functions from one lanthanide metal to another, the magnetic coupling arises from the polarization of the $4 f$ electrons on neighboring lanthanide atoms via the conduction $(6 s)$ electrons. The spin polarization of the conduction electrons is spatially non-uniform and has an oscillatory nature, i.e.

$$
F(x) \propto(x \cos x-\sin x) / x^{4},
$$

where $x=2 k_{F} R$, and $k_{F}$ is the Fermi wave vector and $R$ is the radial distance from a given lanthanide atom. This is schematically shown in Fig. 6, and is known as the Ruderman-KittelKasuya-Yosida (RKKY) interaction. ${ }^{11}$ The interaction between adjacent lanthanide ions will be ferromagnetic when the neighboring lanthanide atom lies in a region of $2 \mathrm{k}_{\mathrm{F}} \mathrm{R}$ where $\mathrm{F}(\mathrm{x})$ is positive, and it will be antiferromagnetic when $\mathrm{F}(\mathrm{x})$ is negative. The strength of the interaction is given by the amplitude of the function $\mathrm{F}(\mathrm{x})$. Because of the lanthanide contraction (i.e. the size of the lanthanide atoms decreases with increasing atomic number), it is possible for the magnetic ordering to change from ferromagnetic to antiferromagnetic in a given $\mathrm{RM}_{\mathrm{x}}$ compound series, provided the sign of $\mathrm{F}(\mathrm{x})$ changes as $2 \mathrm{k}_{\mathrm{F}} \mathrm{R}$ varies from $\mathrm{R}=\mathrm{Ce}$, the first magnetic trivalent lanthanide, to $\mathrm{R}=\mathrm{Yb}$, the last trivalent lanthanide. For example, the $\mathrm{RGe}$ compounds are ferromagnetic for $\mathrm{R}=\mathrm{Ce}, \mathrm{Pr}$, and $\mathrm{Nd}$, while those for $\mathrm{R}=\mathrm{Sm}, \mathrm{Gd}, \mathrm{Tb}, \mathrm{Dy}, \mathrm{Ho}$, and Er are antiferromagnets. $^{11}$

The variation of the RKKY interaction may not play an important of a role in binary intermetallic compounds, especially with respect to cryocooler regenerator materials. However, in ternary alloys it may play a role when substituting one lanthanide $\mathrm{R}^{\prime}$ for another $\mathrm{R}$ [e.g. $\left.\left(R_{1-x} R_{x}^{\prime}\right) M\right]$, or substituting one non-rare-earth metal $M^{\prime}$ for another $M$, [e.g. $R\left(M_{1-x} M_{x}^{\prime}\right)$ ] to adjust the magnetic ordering temperature or the magnetic heat capacity.

\section{IMPROVING REGENERATOR PROPERTIES}

A number of materials have interesting magnetothermal properties for their application as magnetic regenerators, but the ordering temperature is not appropriate for their optimum 


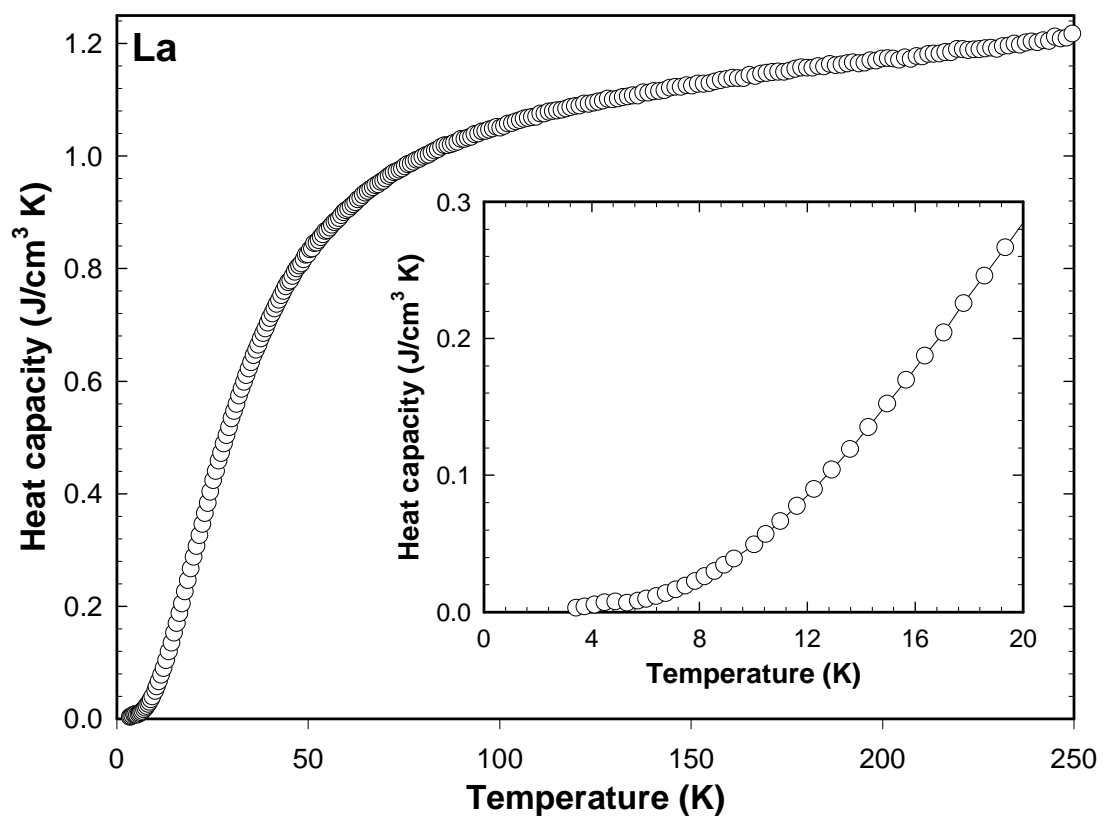

Figure 7. The volumetric heat capacity of La metal from 3 to $250 \mathrm{~K}$. The Debye temperature of La is $150 \mathrm{~K}$.

performance. Recently, we have shown that the magnetothermal properties for Er metal as a replacement for $\mathrm{Pb}$ as a 10 to $50 \mathrm{~K}$ regenerator material was significantly enhanced by alloying with $\operatorname{Pr}$ metal. ${ }^{5,6}$ This has also been done for several binary intermetallic compounds ${ }^{3}$, for example: $\left(\mathrm{Er}_{1-\mathrm{x}} \mathrm{Dy}_{\mathrm{x}}\right) \mathrm{Ni}_{2},\left(\mathrm{Er}_{1-\mathrm{x}} \mathrm{Ho}_{\mathrm{x}}\right) \mathrm{Ni}_{2}, \mathrm{Er}\left(\mathrm{Ni}_{1-\mathrm{x}} \mathrm{Co}_{\mathrm{x}}\right)_{2},\left(\mathrm{Gd}_{0.5} \mathrm{Er}_{0.5}\right) \mathrm{Rh}$, and $\left(\mathrm{Er}_{0.9} \mathrm{Yb}_{0.1}\right) \mathrm{Ni}$.

\section{Lattice Heat Capacity}

One trend that is generally observed in these pseudobinary systems is that the heat capacity maximum increases with increasing ordering temperature. As seen in Fig. 7, the lattice heat capacity increases fairly rapidly with increasing temperature up to $\sim 1 / 2 \Theta$ (where $\Theta$ is the Debye temperature), which is the point where the rate of increase of the heat capacity slows down (i.e. the knee of the heat capacity curve). For example, $\mathrm{ErNi}_{2}$ orders at $6.7 \mathrm{~K}$ and $\mathrm{DyNi}_{2}$ orders at $20.6 \mathrm{~K}$, and the heat capacity maximum increases from $0.42 \mathrm{~J} / \mathrm{cm}^{3} \mathrm{~K}$ for $\mathrm{ErNi}_{2}$ to $0.80 \mathrm{~J} / \mathrm{cm}^{3} \mathrm{~K}$ for $\mathrm{DyNi}_{2}$, see Fig. 8. As one notes the magnetic ordering temperature for intermediate

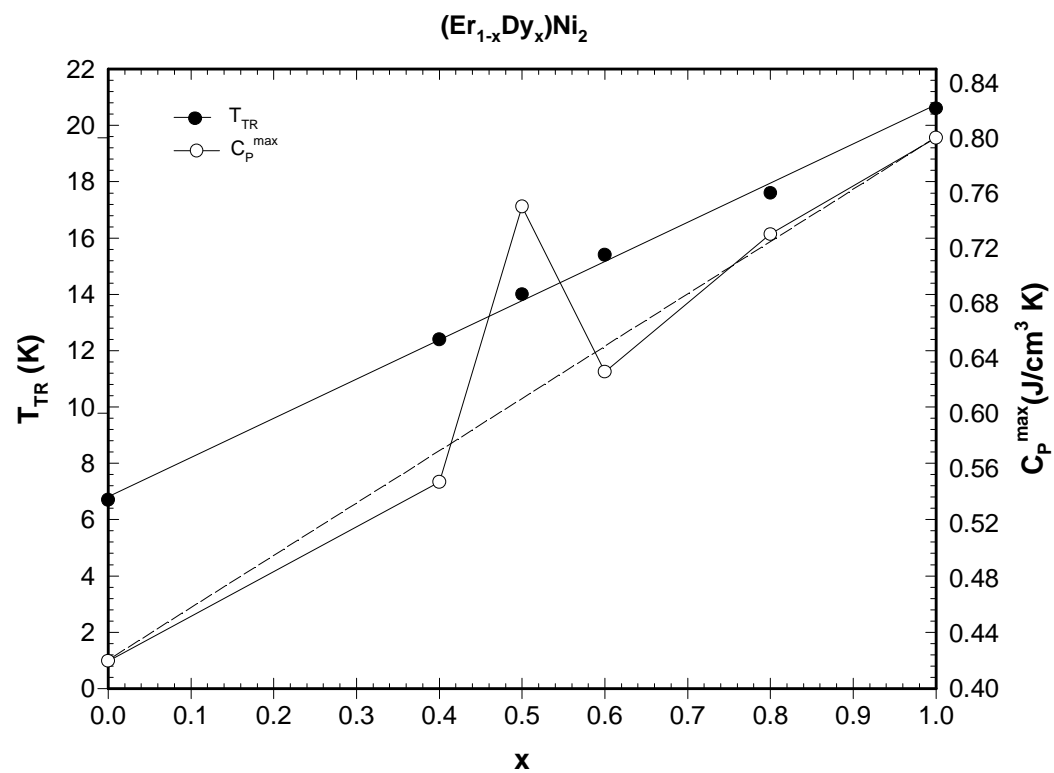

Figure 8. The magnetic ordering temperatures $\left(\mathrm{T}_{\mathrm{TR}}\right)$ and the volumetric heat capacity maxima $\left(\mathrm{C}_{\mathrm{p}}{ }^{\max }\right)$ for the $\left(\mathrm{Er}_{1-\mathrm{x}} \mathrm{Dy}_{\mathrm{x}}\right) \mathrm{Ni}_{2}$ alloys. ${ }^{3}$ 
compositions in the $\left(\mathrm{Er}_{1-\mathrm{x}} \mathrm{Dy} \mathrm{y}_{\mathrm{x}}\right) \mathrm{Ni}_{2}$ system essentially fall on a straight line connecting the two end members, which is consistent with the deGennes factor (see above). The heat capacity maxima increase as expected with increasing magnetic ordering temperature, but two of the data points fall slightly below the straight line connecting the end members, i.e. $\left(\mathrm{Er}_{0.6} \mathrm{Dy}_{0.4}\right) \mathrm{Ni}_{2}$ and $\left(\mathrm{Er}_{0.4} \mathrm{Dy}_{0.6}\right) \mathrm{Ni}_{2}$, while the heat capacity maximum of $\left(\mathrm{Er}_{0.5} \mathrm{Dy}_{0.5}\right) \mathrm{Ni}_{2}$ is significantly higher. Within experimental error the two points which lie below this line may not be anomalous, but the deviation of the $\left(\mathrm{Er}_{0.5} \mathrm{Dy}_{0.5}\right) \mathrm{Ni}_{2}$ value is probably real. At the present time, the reason for the anomaly is unknown, but several possibilities can be ruled out. The magnetic entropies for Er and Dy are equal, see Fig. 1, so a change in $S_{M}$ as a function of $x$ could not account for it. Since $\mathrm{ErNi}_{2}$ and $\mathrm{DyNi}_{2}$ have the same crystal structure (the cubic C15 Laves phase structure), and since the metallic radii of $\operatorname{Er}(1.7566 \AA)$ and Dy $(1.7740 \AA)$ are nearly the same (differ by $1 \%)$, the CEF is essentially the same from $\mathrm{ErNi}_{2}$ to $\mathrm{DyNi}_{2}$ and it is difficult to see how this could cause a change in the magnetic heat capacity peak at just the $\mathrm{x}=0.5$ composition. Similarly, because of the small size difference, it is doubtful that a crystal structure change occurs at $x=0.5$ (e.g. the $\mathrm{x}=0.5$ alloy could possibly have the hexagonal $\mathrm{C} 14$ Laves phase structure), while the other alloys maintain the cubic C15 Laves phase structure. It is possible, however, that the RKKY interaction is such that at $\mathrm{x}=0.5$ the $\mathrm{F}(\mathrm{x})$ value is a maximum or a minimum, and that for $\mathrm{x}=0.4$ and 0.6 the absolute value of $\mathrm{F}(\mathrm{x})$ approaches zero (see Fig. 6) giving rise to the observed behavior, but this would imply a very short period. Clearly, more detailed studies are required to understand this behavior.

\section{The $\left(\operatorname{Er}_{1-\mathrm{x}} \mathbf{Y} \mathbf{b}_{\mathbf{x}}\right) \mathbf{N i}$ System}

The $\left(\operatorname{Er}_{1-\mathrm{x}} \mathrm{Yb}_{\mathrm{x}}\right) \mathrm{Ni}$ system ${ }^{3}$ is quite interesting, since the observed behavior is counter intuitive of what one would expect. ErNi orders at $13.0 \mathrm{~K}$ and has a heat capacity maximum of $0.61 \mathrm{~J} / \mathrm{cm}^{3} \mathrm{~K}$, while for $\mathrm{x}=0.1$ the ordering temperature drops as expected to $9.5 \mathrm{~K}$ on the basis of the deGennes factor, see Fig. 5, but the heat capacity maximum increases by more than $33 \%$ to $0.82 \mathrm{~J} / \mathrm{cm}^{3} \mathrm{~K}$. A significant decrease in heat capacity maximum is expected for two reasons: 1 ) the lattice heat capacity of $\left(\mathrm{Er}_{0.9} \mathrm{Yb}_{0.1}\right) \mathrm{Ni}$ should be smaller because of the lower ordering temperature, and 2) the magnetic entropy of $\mathrm{Yb}$ is significantly smaller than that of $\mathrm{Er}$ (by $\sim 25 \%$ ).

\section{The $\left(\operatorname{Er}_{1-\mathrm{x}} \mathrm{Dy}_{\mathrm{x}}\right) \mathrm{Al}_{2}$ System}

A third example of the occurrence of some unexpected behaviors when a binary compound is alloyed to modify its properties is found in the $\left(\mathrm{Er}_{1-\mathrm{x}} \mathrm{Dy}_{\mathrm{x}}\right) \mathrm{Al}_{2}$ system. ${ }^{12}$ As noted above for the $\left(\mathrm{Er}_{1-\mathrm{x}} \mathrm{Dy}_{\mathrm{x}}\right) \mathrm{Ni}_{2}$ system, the $\mathrm{S}_{\mathrm{M}}$ values of Er and Dy are the same and their metallic radii only differ by about $1 \%$. The two end members and all compositions between them have the cubic C15 Laves phase structure, and the lattice parameters essentially obey Vegard's linear approximation. The magnetic ordering temperature rises in an approximately linear fashion from $13.5 \mathrm{~K}$ for $\mathrm{ErAl}_{2}$ to $63.9 \mathrm{~K}$ for $\mathrm{DyAl}_{2}$, see Fig. 9. From $\sim \mathrm{x}=0.4$ the observed behaviors are as expected, the maximum heat capacity value rises, as does the lattice heat capacity, with increasing $\mathrm{x}$ to pure $\mathrm{DyAl}_{2}(\mathrm{x}=1.0)$. As noted in Fig. 9, the magnetic entropy also increases because the fraction Er decreases and because there is a CEF contribution from $\mathrm{Er}$ at $\sim 8 \mathrm{~K}$ which accounts for $\sim 25 \%$ of $\mathrm{S}_{\mathrm{M}}$, and thus, as the percentage of $\mathrm{Er}$ in the alloy decreases more of the $\mathrm{S}_{\mathrm{M}}$ becomes available for utilization in the magnetic ordering process.

Things, however, become more interesting on the Er-rich side of the $\left(\mathrm{Er}_{1-\mathrm{x}} \mathrm{Dy}_{\mathrm{x}}\right) \mathrm{Al}_{2}$ system. The large heat capacity peak in $\mathrm{ErAl}_{2}$, which is due to a second order paramagnetic to ferromagnetic transition at $13.6 \mathrm{~K}$, drops drastically by more than $50 \%$ when $10 \%$ of the $\mathrm{Er}$ is substituted for by Dy even though the transition temperature rises to $\sim 18 \mathrm{~K}$, see Fig. 9. This behavior is not quite understood. Even more surprising is the existence in $\left(\operatorname{Er}_{0.75} \mathrm{Dy}_{0.25}\right) \mathrm{Al}_{2}$ of a low temperature $(\sim 10 \mathrm{~K})$ first order magnetic transition where the easy axis changes from the $<100\rangle$ for the high temperature phase to the $<111\rangle$ for the low temperature phase, and a high temperature $(\sim 25 \mathrm{~K})$ second order paramagnetic to ferromagnetic transition. ${ }^{11}$ The heat capacity peak maxima are 0.94 and $0.44 \mathrm{~J} / \mathrm{cm}^{3} \mathrm{~K}$, respectively. For higher Dy contents $\mathrm{x} \geq 0.4$ the behavior is normal as noted in the previous paragraph. 


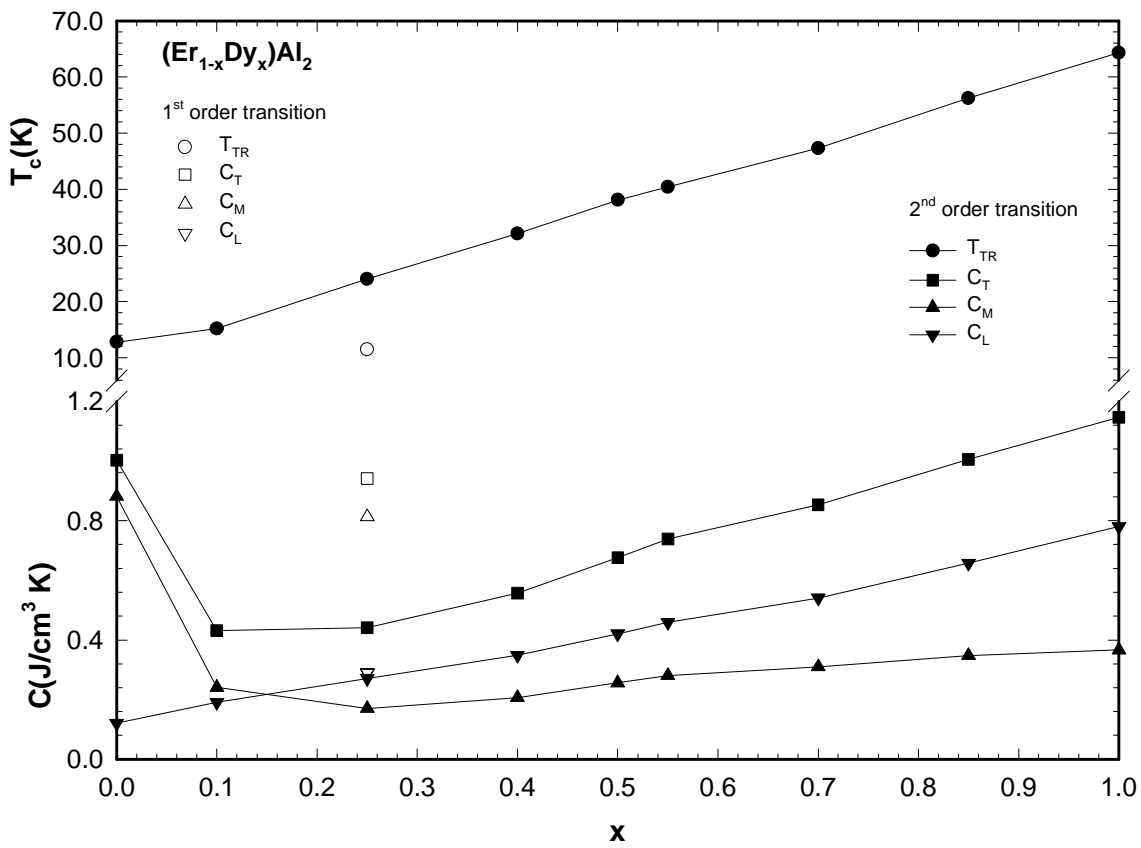

Figure 9. The magnetic ordering temperatures $\left(\mathrm{T}_{T R}\right)$, and the maximum $\left(\mathrm{C}_{T}\right)$, magnetic $\left(\mathrm{C}_{\mathrm{M}}\right)$, and lattice $\left(C_{L}\right)$ volumetric heat capacities for the $\left(\mathrm{Er}_{1-\mathrm{x}} \mathrm{Dy}_{\mathrm{x}}\right) \mathrm{Al}_{2}$ alloys as a function of $\mathrm{x}$.

\section{Non-Rare-Earth Metal Substitutions}

One can also substitute one non-rare-earth metal by another non-rare-earth metal to raise or lower the magnetic ordering temperature, and one similarly expects the heat capacity maximum to increase as the magnetic order temperature increases, just as noted above. However, only limited number of such systems have been investigated. One of them is the $\operatorname{Er}\left(\mathrm{Ni}_{1-\mathrm{x}} \mathrm{Co}_{\mathrm{x}}\right)_{2}$ system. ${ }^{3}$ The magnetic ordering temperature of $\mathrm{ErNi}_{2}$ is $6.7 \mathrm{~K}$ and that of $\mathrm{ErCo}_{2}$ is $33 \mathrm{~K}$. As expected, the volumetric heat capacity of $\mathrm{ErNi}_{2}$ is $0.42 \mathrm{~J} / \mathrm{cm}^{3} \mathrm{~K}$ and that of $\mathrm{ErCo}_{2}$ is $1.60 \mathrm{~J} / \mathrm{cm}^{3} \mathrm{~K}$. However, the behavior of intermediate compositions seem to be somewhat anomalous from the expected behavior. The heat capacity of $\operatorname{Er}\left(\mathrm{Ni}_{0.85} \mathrm{Co}_{0.15}\right)_{2}$ is slightly lower than that of $\mathrm{ErNi}_{2}$, while that of $\operatorname{Er}\left(\mathrm{Ni}_{0.58} \mathrm{Co}_{0.42}\right)_{2}$ is significantly higher as expected, but it still lies below a straight line connecting the two end members, see Fig. 10. This anomalous behavior could be due to CEF effects and/or a change in the RKKY electron polarization, but more detailed work needs to be carried out on these alloys.

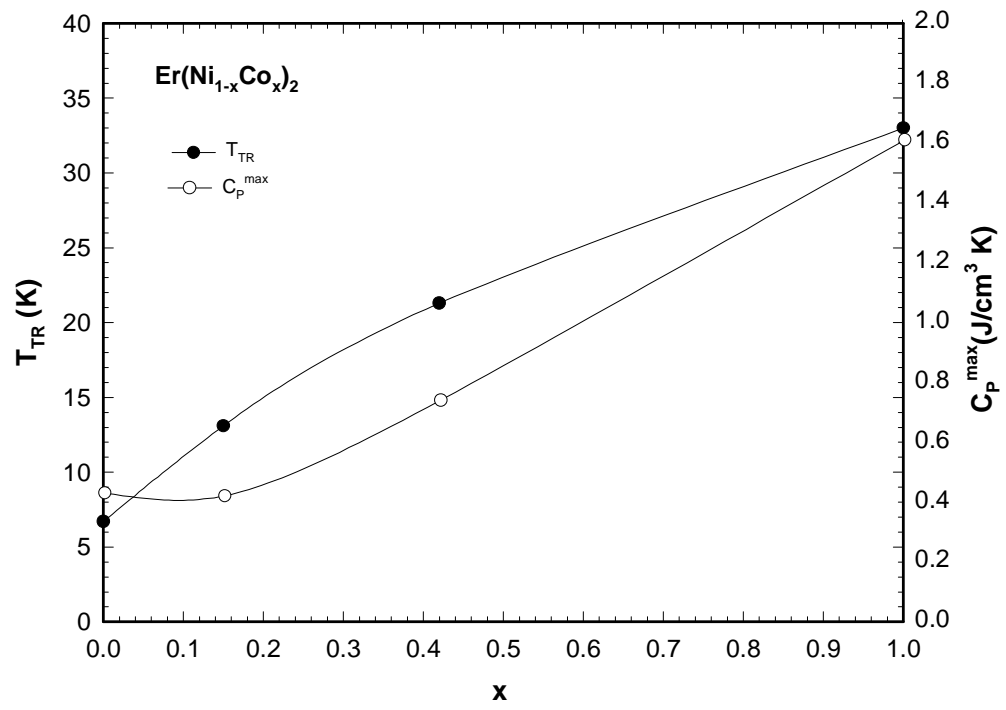

Figure 10. The magnetic ordering temperatures $\left(\mathrm{T}_{\mathrm{TR}}\right)$ and the volumetric heat capacity maxima $\left(\mathrm{C}_{\mathrm{p}}{ }^{\max }\right)$ for the $\operatorname{Er}\left(\mathrm{Ni}_{1-\mathrm{x}} \mathrm{Co}_{\mathrm{x}}\right)_{2}$ alloys. ${ }^{3}$ 


\section{CONCLUDING REMARKS}

The low temperature magnetic heat capacity of a given material can be modified by alloying either a rare-earth metal or a non-rare-earth metal. A number of factors apply and need to be considered when carrying out doping studies (alloying substitutions). These include the theoretical magnetic entropy, the deGennes factor, crystalline electric field effects, the RKKY interaction, and the lattice heat capacity. The third and fourth factors (CEF and RKKY) are difficult to predict a priori and are strongly dependent on the crystal structure of the $\mathrm{RM}_{\mathrm{x}}$ intermetallic compound. Systematic trends are known and reasonably well established but significant and unexpected deviations occur as the alloying agent's concentration is varied. Thus a blending of an Edisonian approach and systematics is necessary to find improved crycooler regenerator materials for applications below $10 \mathrm{~K}$.

\section{ACKNOWLEDGEMENTS}

The applied aspects of this research were sponsored by Atlas Scientific, and the basic research portion by the Office of Basic Energy Sciences, Materials Science Division of the U.S. DOE. The authors also wish to acknowledge the assistance of David Kesse in carrying out some aspects of this work.

\section{REFERENCES}

1. Sahashi, M., Tokai, Y., Kuriyama, T., Nakagome, H., Li, R., Ogawa, M. and Hashimoto, T., "New Magnetic Material R 3 T System with Extremely Large Heat Capacities Used as Heat Regenerators", Adv. Cryogenic Eng., vol. 35 (1990), pp. 1175-1182.

2. Kuriyama, T., Hakamada, R., Nakagome, H., Tokai, Y., Sahashi, M., Li, R., Yoshida, O., Matsumoto, K. and Hashimoto, T., "High Efficient Two-stage GM Refrigerator with Magnetic Material in the Liquid Helium Temperature Region”, Adv. Cryogenic Eng., vol. 35 (1990), pp. 1261-1269.

3. Ackermann, R.A., Cryogenic Regenerative Heat Exchangers, Plenum Press, New York (1997), p. 98.

4. Satoh, T., Onishi, A., Umehara, I., Adachi, Y., Sato, K. and Minehara, E.J., "A Gifford-McMahon Cycle Cryocooler below 2 K" in Cryocoolers 11, Ross, R. G., Jr., ed., Kluwer Academic/Plenum Publishers, New York (2001), pp. 381-386.

5. Gschneidner, K.A., Jr., Pecharsky, A.O. and Pecharsky, V.K., "Ductile, High Heat Capacity, Magnetic Regenerator Alloys for the 10 to $80 \mathrm{~K}$ Temperature Range" in Cryocoolers 11, Ross, R. G., Jr., ed., Kluwer Academic/Plenum Publishers, New York (2001), pp. 433-441.

6. Kashani, A., Helvensteijn, B.P.M., Maddocks, J.R., Kittel, P., Feller, J.R., Gschneidner, K.A., Jr., Pecharsky, V.K. and Pecharsky, A.O., "Performance of a New Regenerator in a Pulse Tube Cooler", Adv. Cryogenic Eng., vol. 47 (2002), pp. 985-991.

7. Kuriyama, T., private communication (March 2001).

8. Bouvier, M., Lethuillier, P. and Schmitt, D., "Specific Heat in Some Gadolinium Compounds. I. Experimental”, Phys. Rev. B, vol. 43 (1991), pp. 13137-13144.

9. Takahashi, A., Tokai, Y., Sahashi, M. and Hashimoto, T., "Specific Heat of a Regenerator Material Er 3 Ni”, Jpn. J. Appl. Phys., vol. 33 (1994), pp. 1023-1026.

10. Nakane, H., Hashimoto, T., Numazawa, T., Okamura, M., Kuriyama, T. and Ohtani, Y., Adv. Cryogenic Eng., vol. 45 (2000), pp. 397-402.

11. Kirchmayr, H.R. and Poldy, C.A., "Magnetic Properties of Intermetallic Compounds of the Rare Earth Metals" in Handbook on the Physics and Chemistry of Rare Earths, Gschneidner, K.A., Jr. and Eyring, L. eds., North-Holland Publishing Co., Amsterdam (1979), Chap. 14, pp. 55-230.

12. Gschneidner, K.A., Jr., Pecharsky, V.K. and Malik, S.K., "The $\left(\mathrm{Dy}_{1-\mathrm{x}} \mathrm{Er}_{\mathrm{x}}\right) \mathrm{Al}_{2}$ Alloys as Active Magnetic Regenerators for Magnetic Refrigeration," Adv. Cryogenics Eng., vol. 42 (1996), pp. 475483. 